\title{
Solutions to Dirichlet-Type Boundary Value Problems of Fractional Order in Banach Spaces
}

\author{
Jing-jing Tan and Cao-zong Cheng \\ College of Applied Sciences, Beijing University of Technology, Beijing 100124, China \\ Correspondence should be addressed to Cao-zong Cheng; czcheng@bjut.edu.cn
}

Received 13 July 2013; Accepted 16 August 2013

Academic Editors: M. Escobedo, G. Mantica, and W. Sun

Copyright (c) 2013 J.-j. Tan and C.-z. Cheng. This is an open access article distributed under the Creative Commons Attribution License, which permits unrestricted use, distribution, and reproduction in any medium, provided the original work is properly cited.

We consider the boundary value problems with Dirichlet-type boundary conditions of nonlinear fractional differential equation in Banach space. The existence of the solution to the boundary value problems is established. Our analysis relies on the Sadovskii fixed point theorem. As an application, we give an example to demonstrate our results.

\section{Introduction}

Fractional differential equations have been of great interest recently. It is caused both by the intensive development of the theory of fractional calculus itself and by the applications in various sciences, such as physics, mechanics, chemistry, and engineering, (e.g., [1-9]).

Consequently, the fractional calculus and its applications in various fields of science and engineering have received much attention and have developed very rapidly. Jiang and Yuan [10], by using fixed point theorem on the cone, discussed the existence and multiplicity of solutions of the nonlinear fractional differential equation boundary value problem as follows:

$$
\begin{gathered}
D_{0+}^{\alpha} u(t)+f(t, u(t))=0, \quad 0<t<1, \\
u(0)=u(1)=0,
\end{gathered}
$$

where $1<\alpha \leq 2$ is a real number and $D_{0+}^{\alpha}$ is standard Riemann-Liouville fractional derivative. The authors in [11] consider the same boundary value problem. They derived the corresponding Green function and obtained the existence of solutions of this problem.

As far as we know, the nonlinear integer order differential equation for the Dirichlet boundary value problem has been studied extensively (e.g., [12-17]). However, only a few papers have dealt with the boundary value problem for fractional differential equation, especially, in Banach spaces. The authors in [18] studied the existence of positive solutions of secondorder two-point boundary value problem as follows:

$$
\begin{gathered}
u^{\prime \prime}(t)+f(t, u(t))=\theta, \quad 0<t<1, \\
u(0)=u(1)=\theta,
\end{gathered}
$$

in Banach spaces. The authors in [19], by using the Mönch fixed point theorems, obtained the same results.

Motivated by the results mentioned above, we discuss the following boundary value problem (BVP for short):

$$
\begin{gathered}
D_{0+}^{\alpha} u(t)+f(t, u(t))=\theta, \quad 0<t<1, \\
u(0)=u(1)=\theta,
\end{gathered}
$$

in Banach space $E$, where $\theta$ is the zero element of $E, 1<$ $\alpha \leq 2$ is a real number, $D_{0+}^{\alpha}$ is standard Riemann-Liouville fractional derivative, $I=[0,1]$, and $f: I \times E \rightarrow E$ is continuous. We establish an existence result of BVP in Banach spaces. The technique relies on the properties of the Kuratowski noncompactness measure and and Sadovskii fixed point theorem. To the best of our knowledge, this is the first paper considering the existence of solutions to Dirichlettype value problems of fractional order in Banach spaces. 


\section{Preliminaries}

For the convenience of the reader, we present here the necessary definitions and preliminary facts which are used throughout this paper.

Definition 1 (see [1]). The Riemann-Liouville fractional integral of order $\alpha>0$ of a function $f:(0, \infty) \rightarrow R$ is given by

$$
I_{0+}^{\alpha} f(x)=\frac{1}{\Gamma(\alpha)} \int_{0}^{x} \frac{f(t)}{(x-t)^{1-\alpha}} d t,
$$

provided that the right side is pointwise defined on $(0, \infty)$, where $\Gamma(\alpha)=\int_{0}^{\infty} t^{\alpha-1} e^{-t} d t$.

Definition 2 (see [1]). The Riemann-liouville fractional derivative of order $\alpha>0$ of a continuous function $f:(0$, $\infty) \rightarrow R$ is given by

$$
D_{0+}^{\alpha} f(x)=\frac{1}{\Gamma(n-\alpha)}\left(\frac{d}{d x}\right)^{n} \int_{0}^{x} \frac{f(t)}{(x-t)^{\alpha-n-1}} d t,
$$

where $n=[\alpha]+1,[\alpha]$ denotes the integer part of the number $\alpha$, provided that the right side is pointwise defined on $(0, \infty)$.

Lemma 3 (see [2]). Let $\alpha>0$. If we assume that $u \in C(0,1) \cap$ $L(0,1)$, then the fractional differential equation

$$
D_{0+}^{\alpha} u(t)=0
$$

has $u(t)=c_{1} t^{\alpha-1}+c_{2} t^{\alpha-2}+\cdots+c_{n} t^{\alpha-N}\left(c_{i} \in R, i=1,2\right.$, $3, \ldots, N)$ as unique solutions, where $N$ is the smallest integer greater than or equal to $\alpha$.

Lemma 4 (see [2]). Assume that $u \in C(0,1) \cap L(0,1)$ with a fractional derivative of order $\alpha>0$ that belongs to $C(0,1) \cap$ $L(0,1)$. Then

$$
I_{0+}^{\alpha} D_{0+}^{\alpha} u(t)=u(t)+c_{1} t^{\alpha-1}+c_{2} t^{\alpha-2}+\cdots+c_{N} t^{\alpha-N},
$$

for some $c_{i} \in R, i=1,2,3, \ldots, N$, where $N$ is the smallest integer greater than or equal to $\alpha$.

Definition 5 (Kuratowski noncompactness measure). Let $E$ be a real Banach space, let $S$ be a bounded set in $E$. We denote

$$
\begin{aligned}
\alpha(S)=\inf \left\{\delta>0: S=\bigcup_{i=1}^{m} S_{i},\right. \\
\left.\quad \operatorname{diam}\left(S_{i}\right)<\delta, i=1,2, \ldots, m\right\} .
\end{aligned}
$$

$\alpha(S)$ is called Kuratowski noncompactness measure of $S$, where $\operatorname{diam}\left(S_{i}\right)$ denote the diameters of $S_{i}$. Obviously $0 \leq$ $\alpha(S)<\infty$.

Definition 6. Let $E_{1}$ and $E_{2}$ be real Banach spaces, $D \subset E_{1}$, and $A: D \rightarrow E_{2}$ be a continuous and bounded operator.
(1) $A$ is called a $k$-set contraction operator if there exists a constant $k$, such that $\alpha(A(S)) \leq k \alpha(S)$ for any bounded set $S$ in $E$. When $k<1, A$ is called a strict set contraction operator.

(2) Let $S$ be a nonrelative compact, bounded subset in $E$. $A$ is called a condensation if $\alpha(A(S))<\alpha(S)$.

Remark 7. A strict set contraction operator is condensation.

Now, we denote the Banach space of continuous functions $u: I \rightarrow E$ by $C[I, E]$ with the maximal norm $\|u\|=\max _{t \in I}\|u(t)\|$. The basic space used in this paper is:

$$
X[I, E]=\left\{u \in C[I, E]: \sup _{t \in I} \frac{\|u(t)\|}{1+t^{\alpha-1}}<+\infty\right\}
$$

equipped with norm $\|u\|_{X}=\sup _{t \in I}\left(\|u(t)\| /\left(1+t^{\alpha-1}\right)\right)$. It is easy to see that $X[I, E]$ is a Banach space.

A map $x \in X[I, E] \cap C^{1}[I, E]$ is called a solution of BVP if it satisfies (3). For a bounded subset $D$ of Banach space $E$, let $\alpha(D)$ be the Kuratowski noncompactness measure of $D$. In this paper, the Kuratowski noncompactness measure in $E$, $C[I, E]$, and $X[I, E]$ is denoted by $\alpha_{E}(\cdot), \alpha_{C}(\cdot)$, and $\alpha_{X}(\cdot)$, respectively. The following properties of the Kuratowski noncompactness measure and Sadovskii fixed point theorem are needed for our discussion.

Lemma 8 (see [20]). If $H \subset C[I, E]$ is bounded and equicontinuous. Then $\alpha_{E}(H(t))$ is continuous on $I$ and $\alpha_{C}(H)=$ $\max _{t \in I} \alpha_{E}(H(t)), \alpha_{E}\left(\int_{I} x(t) d t: x \in H\right) \leq \int_{I} \alpha_{E}(H(t)) d t$, where $H(t)=\{x(t): x \in H\}$ for each $t \in I$.

Lemma 9 (see [21] (Sadovskii)). Let D be a bounded, closed, and convex subset of the Banach space E. If the operator $A$ : $D \rightarrow D$ is condensing, then $A$ has a fixed point in $D$.

\section{Main Result}

In order to discuss the BVP, the preliminary lemmas are given in this section.

For convenience, let us list some conditions.

$\left(H_{1}\right)$ There exist nonnegative functions $a, b \in C[0,1]$ such that

$$
\begin{gathered}
\|f(t, x)\| \leq a(t)\|x\|+b(t), \quad \forall t \in I, \quad x \in E, \\
\int_{0}^{1}\left(1+t^{\alpha-1}\right) a(t) d t<\frac{\Gamma(\alpha)}{2}, \\
\int_{0}^{1} b(t) d t<+\infty .
\end{gathered}
$$

$\left(H_{2}\right)$ For any $r>0,[\alpha, \beta] \subset I, f(t, x)$ is uniformly continuous on $[\alpha, \beta] \times B_{E}[\theta, r]$, where $\theta$ is the zero element of $E$ and $B_{E}[\theta, r]=\{x \in E:\|x\| \leq r\}$.

$\left(H_{3}\right)$ There exists a positive constant $k$ with $(8 / \Gamma(\alpha)(1+$ $\left.\left.t^{\alpha-1}\right)\right) k<1$, such that $\alpha_{E}(f(t, W)) \leq k \alpha_{E}(W)$ for all $t \in[0,1]$ and all bounded subset $W$ in $E$. 
Lemma 10 (see [2]). Given $y \in C[0,1]$ and $1<\alpha \leq 2$, then the problem

$$
\begin{gathered}
D_{0+}^{\alpha} u(t)+y(t)=0, \quad 0<t<1, \\
u(0)=u(1)=0,
\end{gathered}
$$

has a unique solution satisfying

$$
u(t)=-\int_{0}^{t} \frac{(t-s)^{\alpha-1}}{\Gamma(\alpha)} y(s) d s+\int_{0}^{1} \frac{(1-s)^{\alpha-1} t^{\alpha-1}}{\Gamma(\alpha)} y(s) d s
$$

Lemma 11 (see [2]). Suppose that condition $\left(H_{1}\right)$ is satisfied, then BVP is equivalent to integral equation:

$$
\begin{aligned}
u(t)= & -\int_{0}^{t} \frac{(t-s)^{\alpha-1}}{\Gamma(\alpha)} f(s, u(s)) d s \\
& +\int_{0}^{1} \frac{(1-s)^{\alpha-1} t^{\alpha-1}}{\Gamma(\alpha)} f(s, u(s)) d s
\end{aligned}
$$

For any $u \in X[I, E]$, we define the operator $T$ by

$$
\begin{aligned}
(T u)(t)= & -\int_{0}^{t} \frac{(t-s)^{\alpha-1}}{\Gamma(\alpha)} f(s, u(s)) d s \\
& +\int_{0}^{1} \frac{(1-s)^{\alpha-1} t^{\alpha-1}}{\Gamma(\alpha)} f(s, u(s)) d s .
\end{aligned}
$$

Remark 12. Lemma 11 indicates that the solution of BVP coincides with the fixed point of the operator $T . T u$ is well defined and $T u \in X[I, E]$. Indeed, from condition $\left(H_{1}\right)$, we have

$$
\begin{aligned}
& \int_{0}^{1}\|f(t, u(t))\| d t \\
& \quad \leq \int_{0}^{1}[a(t)\|u\|+b(t)] d t \\
& \quad=\int_{0}^{1}\left[a(t)\left(1+t^{\alpha-1}\right) \frac{\|u\|}{1+t^{\alpha-1}}+b(t)\right] d t \\
& \quad \leq \int_{0}^{1}\left[\left(1+t^{\alpha-1}\right) a(t)\|u\|_{X}+b(t)\right] d t \\
& \quad=\|u\|_{X} \int_{0}^{1}\left(1+t^{\alpha-1}\right) a(t) d t+\int_{0}^{1} b(t) d t \\
& \quad<\infty .
\end{aligned}
$$

By (14) and (15), we get that

$$
\begin{aligned}
\left\|\frac{(T u)(t)}{1+t^{\alpha-1}}\right\|= & \frac{1}{1+t^{\alpha-1}} \\
& \times \|-\int_{0}^{t} \frac{(t-s)^{\alpha-1}}{\Gamma(\alpha)} f(s, u(s)) d s \\
& \quad+\int_{0}^{1} \frac{(1-s)^{\alpha-1} t^{\alpha-1}}{\Gamma(\alpha)} f(s, u(s)) d s \| \\
\leq & \frac{2}{\Gamma(\alpha)} \int_{0}^{1}\|f(s, u(s))\| d s \\
& <\infty .
\end{aligned}
$$

Lemma 13. Suppose conditions $\left(H_{1}\right)$ and $\left(H_{2}\right)$ are satisfied, then $T: X[I, E] \rightarrow X[I, E]$ is continuous and bounded.

Proof. First, by (14) and (16), we get $(T u)(t) \in X[I, E]$ for any $u(t) \in X[I, E]$. Thus, $T: X[I, E] \rightarrow X[I, E]$ is bounded. Next, we will prove that $T$ is continuous on $X[I, E]$. Take $\left\{u_{n}\right\}$, $\{u\} \in X[I, E]$ and $\left\|u_{n}-u\right\|_{X} \rightarrow 0(n \rightarrow+\infty)$. Hence, $\left\{u_{n}\right\}$ is a bounded subset of $X[I, E]$, that is, there exists $M>0$ such that $\left\|u_{n}\right\|_{X} \leq M$ for all $n \geq 1$. Taking the limit, we have $\|u\|_{X} \leq M$.

In addition, by (14) we have

$$
\begin{aligned}
& \left\|\frac{\left(T u_{n}\right)(t)}{1+t^{\alpha-1}}-\frac{(T u)(t)}{1+t^{\alpha-1}}\right\| \\
& =\| \frac{1}{1+t^{\alpha-1}}\left[-\int_{0}^{t} \frac{(t-s)^{\alpha-1}}{\Gamma(\alpha)} f\left(s, u_{n}(s)\right) d s\right. \\
& \left.+\int_{0}^{1} \frac{(1-s)^{\alpha-1} t^{\alpha-1}}{\Gamma(\alpha)} f\left(s, u_{n}(s)\right) d s\right] \\
& -\frac{1}{1+t^{\alpha-1}}\left[-\int_{0}^{t} \frac{(t-s)^{\alpha-1}}{\Gamma(\alpha)} f(s, u(s)) d s\right. \\
& \left.+\int_{0}^{1} \frac{(1-s)^{\alpha-1} t^{\alpha-1}}{\Gamma(\alpha)} f(s, u(s)) d s\right] \| \\
& \leq \| \frac{1}{1+t^{\alpha-1}}\left[-\int_{0}^{t} \frac{(t-s)^{\alpha-1}}{\Gamma(\alpha)} f\left(s, u_{n}(s)\right) d s\right. \\
& \left.+\int_{0}^{t} \frac{(t-s)^{\alpha-1}}{\Gamma(\alpha)} f(s, u(s)) d s\right] \| \\
& +\| \frac{1}{1+t^{\alpha-1}}\left[\int_{0}^{1} \frac{(1-s)^{\alpha-1} t^{\alpha-1}}{\Gamma(\alpha)} f\left(s, u_{n}(s)\right) d s\right. \\
& \left.-\int_{0}^{1} \frac{(1-s)^{\alpha-1} t^{\alpha-1}}{\Gamma(\alpha)} f(s, u(s)) d s\right] \| \\
& \leq \frac{2}{\Gamma(\alpha)\left(1+t^{\alpha-1}\right)} \int_{0}^{1}\left\|f\left(s, u_{n}(s)\right)-f(s, u(s))\right\| d s .
\end{aligned}
$$


It follows from condition $\left(H_{2}\right)$ that for any $\varepsilon>0$, there exists $N>0$ such that

$$
\left\|f\left(s, u_{n}(s)\right)-f(s, u(s))\right\| \leq \frac{\varepsilon}{3} \Gamma(\alpha), \quad \forall n \geq N, s \in I .
$$

Therefore, for any $\varepsilon>0, t \in[0,1]$, and $n>N$, by (17) and (18), we have

$$
\left\|\frac{\left(T u_{n}\right)(t)}{1+t^{\alpha-1}}-\frac{(T u)(t)}{1+t^{\alpha-1}}\right\| \leq \frac{2}{\Gamma(\alpha)\left(1+t^{\alpha-1}\right)} \int_{0}^{1} \frac{\varepsilon}{3} \Gamma(\alpha) d s<\varepsilon .
$$

Thus, we conclude that $\left\|T u_{n}-T u\right\|_{X}<\varepsilon$; namely, $T: X[I$, $E] \rightarrow X[I, E]$ is continuous, and the conclusion of lemma follows.

Lemma 14. Let condition $\left(H_{1}\right)$ be satisfied and let $V$ be a bounded subset of $X[I, E]$. Then $(T V)(t) /\left(1+t^{\alpha-1}\right)$ is equicontinuous on $[0,1]$.

Proof. In order to show that $(T V)(t) /\left(1+t^{\alpha-1}\right)$ is equicontinuous on $[0,1]$, we only need to testify the following conclusion.

For any $\varepsilon>0, u \in V$, there exists a $\delta>0$ such that

$$
\begin{array}{r}
\left\|\frac{(T u)\left(t_{2}\right)}{1+t_{2}^{\alpha-1}}-\frac{(T u)\left(t_{1}\right)}{1+t_{1}^{\alpha-1}}\right\|<\varepsilon, \quad \forall t_{1}, t_{2} \in[0,1], \\
\left|t_{1}-t_{2}\right|<\delta .
\end{array}
$$

In fact, from condition $\left(H_{1}\right)$, it follows that

$\left(a_{1}\right)$ nonnegative functions $a(t), b(t) \in C[0,1]$ are bounded in $[0,1]$, that is, there exist positive constants $A$ and $B$, such that $\max _{t \in I} a(t) \leq A$ and $\max _{t \in I} b(t) \leq B$,

$\left(a_{2}\right)\|f(t, u(t))\| \leq\left(1+t^{\alpha-1}\right) a(t)\|u\|_{X}+b(t)$.

In view of the boundedness of $V$, there exists $R>0$ such that $\|u\|_{X} \leq R$ for any $u \in V$. Without loss of generality, for any $u \in V, t_{1}, t_{2} \in I$ with $t_{1}<t_{2}$, by (14), we can find that

$$
\begin{gathered}
\left\|\frac{(\mathrm{Tu})\left(t_{2}\right)}{1+t_{2}^{\alpha-1}}-\frac{(\mathrm{Tu})\left(t_{1}\right) \|}{1+t_{1}^{\alpha-1}}\right\| \\
=\|-\int_{0}^{t_{2}} \frac{\left(t_{2}-s\right)^{\alpha-1}}{\left(1+t_{2}^{\alpha-1}\right) \Gamma(\alpha)} f(s, u(s)) d s \\
+\int_{0}^{1} \frac{(1-s)^{\alpha-1} t_{2}^{\alpha-1}}{\left(1+t_{2}^{\alpha-1}\right) \Gamma(\alpha)} f(s, u(s)) d s \\
-\left[-\int_{0}^{t_{1}} \frac{\left(t_{1}-s\right)^{\alpha-1}}{\left(1+t_{1}^{\alpha-1}\right) \Gamma(\alpha)} f(s, u(s)) d s\right.
\end{gathered}
$$

$$
\begin{aligned}
& \left.+\int_{0}^{1} \frac{(1-s)^{\alpha-1} t_{1}^{\alpha-1}}{\left(1+t_{1}^{\alpha-1}\right) \Gamma(\alpha)} f(s, u(s)) d s\right] \| \\
& \leq \| \int_{0}^{t_{2}} \frac{\left(t_{2}-s\right)^{\alpha-1}}{\left(1+t_{2}^{\alpha-1}\right) \Gamma(\alpha)} f(s, u(s)) d s \\
& -\int_{0}^{t_{1}} \frac{\left(t_{1}-s\right)^{\alpha-1}}{\left(1+t_{1}^{\alpha-1}\right) \Gamma(\alpha)} f(s, u(s)) d s \| \\
& +\| \int_{0}^{1} \frac{(1-s)^{\alpha-1} t_{2}^{\alpha-1}}{\left(1+t_{2}^{\alpha-1}\right) \Gamma(\alpha)} f(s, u(s)) d s \\
& -\int_{0}^{1} \frac{(1-s)^{\alpha-1} t_{1}^{\alpha-1}}{\left(1+t_{1}^{\alpha-1}\right) \Gamma(\alpha)} f(s, u(s)) d s \| \\
& \leq \frac{1}{\Gamma(\alpha)} \| \int_{0}^{t_{2}} \frac{\left(t_{2}-s\right)^{\alpha-1}}{1+t_{2}^{\alpha-1}} f(s, u(s)) d s \\
& -\int_{0}^{t_{1}} \frac{\left(t_{2}-s\right)^{\alpha-1}}{1+t_{2}^{\alpha-1}} f(s, u(s)) d s \| \\
& +\frac{1}{\Gamma(\alpha)} \| \int_{0}^{t_{1}} \frac{\left(t_{2}-s\right)^{\alpha-1}}{1+t_{2}^{\alpha-1}} f(s, u(s)) d s \\
& -\int_{0}^{t_{1}} \frac{\left(t_{1}-s\right)^{\alpha-1}}{1+t_{1}^{\alpha-1}} f(s, u(s)) d s \| \\
& +\frac{1}{\Gamma(\alpha)} \| \int_{0}^{1}\left[\frac{t_{2}^{\alpha-1}}{1+t_{2}^{\alpha-1}}-\frac{t_{1}^{\alpha-1}}{1+t_{1}^{\alpha-1}}\right] \\
& \times(1-s)^{\alpha-1} f(s, u(s)) d s \|
\end{aligned}
$$$$
\leq \frac{1}{\Gamma(\alpha)} \int_{t_{1}}^{t_{2}} \frac{\left(t_{2}-s\right)^{\alpha-1}}{1+t_{2}^{\alpha-1}}\|f(s, u(s))\| d s
$$$$
+\frac{1}{\Gamma(\alpha)} \int_{0}^{t_{1}}\left[\frac{\left(t_{2}-s\right)^{\alpha-1}}{1+t_{2}^{\alpha-1}}-\frac{\left(t_{1}-s\right)^{\alpha-1}}{1+t_{1}^{\alpha-1}}\right]
$$$$
\times\|f(s, u(s))\| d s
$$$$
+\frac{1}{\Gamma(\alpha)} \int_{0}^{1}\left[\frac{t_{2}^{\alpha-1}}{1+t_{2}^{\alpha-1}}-\frac{t_{1}^{\alpha-1}}{1+t_{1}^{\alpha-1}}\right](1-s)^{\alpha-1}
$$$$
\times\|f(s, u(s))\| d s
$$$$
\leq \frac{2 A R+B}{\Gamma(\alpha)}\left(t_{2}-t_{1}\right)
$$$$
+\frac{2 A R+B}{\Gamma(\alpha+1)}\left[\frac{t_{2}^{\alpha}}{1+t_{2}^{\alpha-1}}-\frac{t_{1}^{\alpha}}{1+t_{1}^{\alpha-1}}-\frac{\left(t_{2}-t_{1}\right)^{\alpha}}{2}\right]
$$$$
+\frac{2 A R+B}{\Gamma(\alpha)}\left(\frac{t_{2}^{\alpha-1}}{1+t_{2}^{\alpha-1}}-\frac{t_{1}^{\alpha-1}}{1+t_{1}^{\alpha-1}}\right) \text {. }
$$ 
This ensures that $(T V)(t) /\left(1+t^{\alpha-1}\right)$ is equicontinuous on $[0,1]$. If $t_{1} \geq t_{2}$, we can also get the same result. Thus, $(T V)(t) /\left(1+t^{\alpha-1}\right)$ is equicontinuous on $[0,1]$.

The main result of this paper is as follows.

Theorem 15. Let conditions $\left(H_{1}\right)-\left(H_{3}\right)$ be satisfied. Then the $B V P$ has at least one solution belonging to $X[I, E]$.

Proof. We only need to prove that the the operator $T$ has a fixed point in $X[I, E]$. By condition $\left(H_{1}\right)$, we can choose a real number $R$ such that

$$
R>\int_{0}^{1} b(s) d s\left[\frac{\Gamma(\alpha)}{2}-\int_{0}^{1}\left(1+s^{\alpha-1}\right) a(s) d s\right]^{-1},
$$

and let

$$
B=: B_{X}(\theta, R)=\left\{u \in X[I, E]:\|u\|_{X} \leq R\right\} .
$$

Frist we prove that $T B \subset B$. In fact, for any $u \in B$, by (16), we have

$$
\begin{aligned}
& \left\|\frac{(T u)(t)}{1+t^{\alpha-1}}\right\| \\
& \leq \frac{2}{\Gamma(\alpha)} \int_{0}^{1}\|f(s, u(s))\| d s \\
& \leq \frac{2}{\Gamma(\alpha)}\left\{\int_{0}^{1}\left[\left(1+s^{\alpha-1}\right) a(s)\right] d s\|u\|_{X}+\int_{0}^{1} b(s) d s\right\} \\
& \leq \frac{2}{\Gamma(\alpha)}\left\{\int_{0}^{1}\left[\left(1+s^{\alpha-1}\right) a(s)\right] d s R\right. \\
& \left.\quad+R\left[\frac{\Gamma(\alpha)}{2}-\int_{0}^{1}\left(1+s^{\alpha-1}\right) a(s) d s\right]\right\}
\end{aligned}
$$$$
<R, \quad(\forall t \in I)
$$

From Lemma 13, it follows that $T B \subset B$.

Choose $D=\overline{c o}_{X}(T B)$, that is, $D$ is the convex closure of $T B$ in $X[I, E]$. Clearly, $D$ is nonempty, bounded, convex, and closed subset of $B$. By Lemma 14, it follows that $(T B)(t) /\left(1+t^{\alpha-1}\right)$ is equicontinuous on $[0,1]$, together with the definition of $D$, it follows that $D(t) /\left(1+t^{\alpha-1}\right)$ are equicontinuous on $[0,1]$.

Now we show that $T$ is a strict set contraction operator from $D$ to $D$.

Observing that $D \subset B$ and $T B \subset D$, together with Lemma 13 we know that $T: D \rightarrow D$ is bounded and continuous. Finally, we prove that there exists a constant $0 \leq$ $a<1$ such that $\alpha_{X}(T V) \leq a \alpha_{X}(V)$, for $V \subset D$.

In fact, by (14), condition $\left(H_{2}\right)$ and Lemma 14, applying [22, Lemma 2.6], we have

$$
\alpha_{X}(T V)=\sup _{t \in I} \alpha_{E}\left(\frac{(T V)(t)}{1+t^{\alpha-1}}\right), \quad \forall V \subset D,
$$

where $(T V)(t) /\left(1+t^{\alpha-1}\right)=\left\{(T u)(t) /\left(1+t^{\alpha-1}\right): u \in V\right.$, $t$ is fixed $\subset \subset D$ for each $t \in I$. Thus, by (25), we need only to prove that $\sup _{t \in I} \alpha_{E}\left((T u)(t) /\left(1+t^{\alpha-1}\right)\right) \leq a \alpha_{X}(V)$, for $V \subset D$. By condition $\left(H_{2}\right)$ and the definition of $D$, we know $\{f(s, u(s)): u \in D\}$ are equicontinuous on $[0,1]$. Thus, by virtue of Lemma 8 and condition $\left(\mathrm{H}_{3}\right)$, we get

$$
\begin{aligned}
\alpha_{E}\left(\frac{(T V)(t)}{1+t^{\alpha-1}}\right) \\
\leq-\frac{1}{1+t^{\alpha-1}} \int_{0}^{t} \frac{(t-s)^{\alpha-1}}{\Gamma(\alpha)} \alpha_{E}(\{f(s, u(s)): u \in V\}) d s \\
\quad+\frac{1}{1+t^{\alpha-1}} \int_{0}^{1} \frac{(1-s)^{\alpha-1} t^{\alpha-1}}{\Gamma(\alpha)} \\
\leq \frac{2}{\Gamma(\alpha)\left(1+t^{\alpha-1}\right)} \int_{0}^{1} \alpha_{E}(\{f(s, u(s)): u \in V\}) d s \\
=\frac{2}{\Gamma(\alpha)\left(1+t^{\alpha-1}\right)} \int_{0}^{1} \alpha_{E}(f(s, W)) d s \\
\leq \frac{2}{\Gamma(\alpha)\left(1+t^{\alpha-1}\right)} k \alpha_{E}(W),
\end{aligned}
$$

where $W=\{u(s): s \in I, u \in V\}$. For any given $\varepsilon>0$, there exists a partition $V=\bigcup_{j=1}^{n} V_{j}$ with

$$
\operatorname{diam}\left(V_{j}\right)<\alpha_{X}(V)+\frac{\varepsilon}{5}, \quad j=1,2, \ldots, n .
$$

Now for $j=1,2, \ldots, n$, choose $u_{j} \in V_{j}$ and a partition $0=$ $t_{0}<t_{1}<t_{2}<\cdots<t_{m}=1$ such that

$$
\begin{array}{r}
\left\|u_{j}(t)-u_{j}(\bar{t})\right\|<\frac{\varepsilon}{5}, \quad \forall j=1,2, \ldots, n, \\
t, \bar{t} \in\left[t_{i-1}, t_{i}\right], \quad \forall i=1,2, \ldots, m .
\end{array}
$$

Clearly, $W=\bigcup_{i=1}^{m} \bigcup_{j=1}^{n} W_{i j}$, where $W_{i j}=\left\{u(t): t \in\left[t_{i-1}, t_{i}\right]\right.$, $\left.u \in V_{j}\right\}$. For any $u(t), \bar{u}(\bar{t}) \in W_{i j}\left(t, \bar{t} \in\left[t_{i-1}, t_{i}\right], u, \bar{u} \in V_{j}\right)$, by (27) and (28), we have

$$
\begin{aligned}
\|u(t)-\bar{u}(\bar{t})\| & \\
\leq & \left\|u(t)-u_{j}(t)\right\|+\left\|u_{j}(t)-u_{j}(\bar{t})\right\|+\left\|u_{j}(\bar{t})-\bar{u}(\bar{t})\right\| \\
= & \left(1+t^{\alpha-1}\right) \frac{\left\|u(t)-u_{j}(t)\right\|}{1+t^{\alpha-1}} \\
& +\left\|u_{j}(t)-u_{j}(\bar{t})\right\|+\left(1+\bar{t}^{\alpha-1}\right) \frac{\left\|u_{j}(\bar{t})-\bar{u}(\bar{t})\right\|}{1+\bar{t}^{\alpha-1}} \\
\leq & \left(1+t^{\alpha-1}\right)\left\|u-u_{j}\right\|_{X}+\frac{\varepsilon}{5}+\left(1+\bar{t}^{\alpha-1}\right)\left\|u_{j}-\bar{u}\right\|_{X} \\
\leq & 4 \operatorname{diam}\left(V_{j}\right)+\frac{\varepsilon}{5} \\
< & 4 \alpha_{X}(V)+\varepsilon,
\end{aligned}
$$


which implies diam $W_{i j} \leq 4 \alpha_{X}(V)+\varepsilon$ and, thus, $\alpha_{E}(W) \leq$ $4 \alpha_{X}(V)+\varepsilon$. Since $\varepsilon$ is arbitrary, we get

$$
\alpha_{E}(W) \leq 4 \alpha_{X}(V) .
$$

It follows from (25), (26), and (30) that

$$
\alpha_{E}\left(\frac{(T V)(t)}{1+t^{\alpha-1}}\right) \leq \frac{8}{\Gamma(\alpha)\left(1+t^{\alpha-1}\right)} k \alpha_{X}(V), \quad \forall V \subset D .
$$

Thus

$$
\sup _{t \in I} \alpha_{E}\left(\frac{(T V)(t)}{1+t^{\alpha-1}}\right) \leq \frac{8}{\Gamma(\alpha)\left(1+t^{\alpha-1}\right)} k \alpha_{X}(V), \quad \forall V \subset D .
$$

Consequently

$$
\alpha_{X}(T V) \leq a \alpha_{X}(V),
$$

where $a=\left(8 / \Gamma(\alpha)\left(1+t^{\alpha-1}\right)\right) k$. From condition $\left(H_{3}\right)$, it follows that $0 \leq a<1$. Therefore, $T$ is a strict set contraction operator from $D$ to $D$, obviously $T$ is condensing too. It follows from Lemma 9 that $T$ has at least one fixed point in $D$, that is, the BVP has at least one solution in $X[I, E]$.

\section{Example}

Now we consider the system of scalar nonlinear fractional differential equations to illustrate our results.

Let

$$
\begin{aligned}
E=l_{\infty}=\{u & =\left(u_{1}, u_{2}, \ldots, u_{n}, \ldots\right), \\
& \left.\sup _{n}\left|u_{n}\right|<+\infty, \quad t \in[0,1]\right\},
\end{aligned}
$$

with the norm $\|u\|=\sup _{n}\left|u_{n}\right|$. Evidently $E$ is a Banach space. Consider the boundary value problem:

$$
\begin{gathered}
D^{3 / 2} u_{n}=\frac{u_{n}}{16(1+\sqrt{t})\left(1+t^{2}\right)}+\frac{\sin t+\sqrt{\left|\sin u_{n+1}^{2}\right|}}{42 n^{2} e^{\sqrt{t}}}, \\
t \in(0,1), \\
u_{n}(0)=u_{n}(1)=0 .
\end{gathered}
$$

System (35) can be regard as a boundary value problem of the form (3), where

$$
\begin{gathered}
\alpha=\frac{3}{2}, \\
f(t, u)=\left(f_{1}(t, u), f_{2}(t, u), \ldots, f_{n}(t, u), \ldots\right) \\
\text { with } f_{n}(t, u)=\frac{u_{n}}{16(1+\sqrt{t})\left(1+t^{2}\right)}+\frac{\sin t+\sqrt{\left|\sin u_{n+1}^{2}\right|}}{42 n^{2} e^{\sqrt{t}}} .
\end{gathered}
$$

Next, we show that conditions $\left(H_{1}\right)-\left(H_{3}\right)$ are satisfied. Clearly, $f(t, u) \in C(I \times E, E)$ and

$$
\|f(t, u)\| \leq\left[\frac{1}{16(1+\sqrt{t})\left(1+t^{2}\right)}+\frac{1}{42 e^{\sqrt{t}}}\right]\|u\|+\frac{1}{42 e^{\sqrt{t}}} .
$$

With the aid of simple computation, we have

$$
\begin{gathered}
\int_{0}^{1}(1+\sqrt{t})\left[\frac{1}{16(1+\sqrt{t})\left(1+t^{2}\right)}+\frac{1}{42 e^{\sqrt{t}}}\right] d t \\
=\frac{\pi}{64}+\frac{1}{7}-\frac{1}{3 e} \approx 0.0693 \\
\int_{0}^{1} \frac{1}{42 e^{\sqrt{t}}} d t \approx 0.0126 .
\end{gathered}
$$

Note that $\Gamma(3 / 2) \approx 0.8862$. Take $a(t)=(1 / 16(1+\sqrt{t})(1+$ $\left.\left.t^{2}\right)\right)+\left(1 / 42 e^{\sqrt{t}}\right)$ and $b(t)=1 / 42 e^{\sqrt{t}}$. Clearly, condition $\left(H_{1}\right)$ is satisfied. It is easy to see that condition $\left(H_{2}\right)$ is also satisfied.

Finally, we verify condition $\left(H_{3}\right)$. Denote $f=f^{(1)}+f^{(2)}$, where

$$
\begin{aligned}
f_{n}^{(1)} & =\frac{u_{n}(t)}{16(1+\sqrt{t})\left(1+t^{2}\right)}, \\
f_{n}^{(2)} & =\frac{\sin t+\sqrt{\left|\sin u_{n+1}^{2}(t)\right|}}{42 n^{2} e^{\sqrt{t}}} .
\end{aligned}
$$

Then we can obtain that $\alpha\left(f^{(2)}(t, D)\right)=0$ for any bounded set $D \subset E$. Indeed, let $\left\{u^{(m)}\right\} \subset E$ be bounded, that is, there exists $M>0$, such that $\left\|u^{(m)}\right\| \leq M, m=1,2,3, \ldots$, where $u^{(m)}=\left(u_{1}^{(m)}, \ldots, u_{n}^{(m)}, \ldots\right)$. Then we have, for each $t \in$ I,

$$
\left\|f_{n}^{(2)}\left(t, u^{(m)}\right)\right\| \leq \frac{1+M}{42 n^{2}}, \quad n, m=1,2,3, \ldots
$$

which implied that $\left\{f_{n}^{(2)}\left(t, u^{(m)}\right)\right\}$ is bounded. And by the diagonal method, we can choose a subsequence $\left\{u^{\left(m_{k}\right)}\right\} \subset$ $\left\{u^{(m)}\right\}$ such that

$$
f_{n}^{(2)}\left(t, u^{\left(m_{k}\right)}\right) \longrightarrow v_{n}, \quad k \longrightarrow \infty, n=1,2,3, \ldots
$$

From (40), it follows that

$$
\left\|v_{n}\right\| \leq \frac{1+M}{42 n^{2}}, \quad n=1,2,3, \ldots
$$

that is, $v=\left\{v_{1}, \ldots, v_{n}, \ldots\right\} \in E$.

For any $\varepsilon>0,(40)$ and (42) imply that there exists $N>$ 0 such that

$$
\left\|f_{n}^{(2)}\left(t, u^{\left(m_{k}\right)}\right)\right\|<\frac{\varepsilon}{2}, \quad\left\|v_{n}\right\|<\frac{\varepsilon}{2}, \quad n>N, k=1,2,3, \ldots
$$

On the other hand, from (41) we obtain that there exists $K$ > 0 such that

$$
\left\|f_{n}^{(2)}\left(t, u^{\left(m_{k}\right)}\right)-v_{n}\right\|<\varepsilon, \quad k>K, n=1,2, \ldots N .
$$


It follows from (43), (44), and the definition of the norm in $E$ that

$$
\left\|f^{(2)}\left(t, u^{\left(m_{k}\right)}\right)-v\right\|<\varepsilon, \quad k>K .
$$

This means that $\left\|f^{(2)}\left(t, u^{\left(m_{k}\right)}\right)-v\right\| \rightarrow 0$ as $k \rightarrow \infty$ and so $f^{(2)}(t, D)$ is relatively compact for any bounded $D \subset E$. Hence

$$
\alpha\left(f^{(2)}(t, D)\right)=0, \quad \forall t \in I, D \subset E .
$$

Consequently, we arrive at

$$
\begin{aligned}
\alpha(f(t, D)) & \leq \alpha\left(f^{(1)}(t, D)\right) \\
& =\frac{\alpha(D)}{16(1+\sqrt{t})\left(1+t^{2}\right)} \\
& \leq \frac{1}{16} \alpha(D), \quad \forall t \in I, D \subset E,
\end{aligned}
$$

since

$$
\frac{8}{(1+\sqrt{t}) \Gamma(2 / 3)} \frac{1}{16}<1
$$

we conclude that condition $\left(H_{3}\right)$ is satisfied for $k=1 / 16$.

Therefore, all the conditions of Theorem 15 are satisfied. An application of Theorem 15 implies that problem (35) has a solution.

\section{Conclusion}

In this paper, we present some sufficient conditions which ensure the existence of solutions to fractional differential equation for Dirichlet-type boundary value problems. Applying the Sadovskii fixed point theorem, we establish some new existence criteria for boundary value problems (3) in Banach space. Although, for the fractional differential equation for the Dirichlet boundary value problem (3), only a few papers have dealt with the boundary value problem for fractional differential equations, especially in Banach space. In this aspect, our work fills up the deficiency. As applications, examples are presented to illustrate the main results.

\section{Conflict of Interests}

The authors declare that there is no conflict of interests regarding the publication of this paper.

\section{References}

[1] S. G. Samko, A. A. Kilbas, and O. I. Marichev, Fractional Integral and Derivatives, Theory and Applications, Gordon and Breach, Yverdon, Switzerland, 1993.

[2] Z. Bai and H. Lü, "Positive solutions for boundary value problem of nonlinear fractional differential equation," Journal of Mathematical Analysis and Applications, vol. 311, no. 2, pp. 495-505, 2005.
[3] M. A. Krasnosel'skii, Positive Solutions of Operator Equations, Noordhoff, Groningen, The Netherlands, 1964.

[4] V. Lakshmikantham and A. S. Vatsala, "General uniqueness and monotone iterative technique for fractional differential equations," Applied Mathematics Letters, vol. 21, no. 8, pp. 828 834, 2008.

[5] X. Lin and D. Jiang, "Multiple positive solutions of Dirichlet boundary value problems for second order impulsive differential equations," Journal of Mathematical Analysis and Applications, vol. 321, no. 2, pp. 501-514, 2006.

[6] K. S. Miller and B. Ross, An Introdction to the Fractional Calculus and Fractional Differential Equations, John Wiley \& Sons, New York, NY, USA, 1993.

[7] I. Podlubny, Fractional Differential Equations, Mathematics in Science and Engineering, Academic Press, New York, NY, USA, 1999.

[8] D. Guo, V. Lakshmikantham, and X. Liu, Nonlinear Integral Equations in Abstract Spaces, Kluwer Academic, Dordrecht, The Netherlands, 1996.

[9] V. Lakshmikantham and S. Leela, Nonlinear Differential Equations in Abstract Spaces, Pergamon Press, Oxford, UK, 1981.

[10] D. Jiang and C. Yuan, "The positive properties of the Green function for Dirichlet-type boundary value problems of nonlinear fractional differential equations and its application," Nonlinear Analysis: Theory, Methods \& Applications A, vol. 72, no. 2, pp. 710-719, 2010.

[11] V. Lakshmikantham and A. S. Vatsala, "Basic theory of fractional differential equations," Nonlinear Analysis: Theory, Methods \& Applications A, vol. 69, no. 8, pp. 2677-2682, 2008.

[12] Z. Wei, "Positive solution of singular Dirichlet boundary value problems for second order differential equation system," Journal of Mathematical Analysis and Applications, vol. 328, no. 2, pp. 1255-1267, 2007.

[13] X. Zhang, L. Liu, and Y. Wu, "Positive solutions of nonresonance semipositone singular Dirichlet boundary value problems," Nonlinear Analysis: Theory, Methods \& Applications A, vol. 68, no. 1, pp. 97-108, 2008.

[14] X. Xu, D. Jiang, and C. Yuan, "Multiple positive solutions to singular positone and semipositone Dirichlet-type boundary value problems of nonlinear fractional differential equations," Nonlinear Analysis: Theory, Methods \& Applications A, vol. 74, no. 16, pp. 5685-5696, 2011.

[15] B. Liu, "Positive solutions of a nonlinear four-point boundary value problems in Banach spaces," Journal of Mathematical Analysis and Applications, vol. 305, no. 1, pp. 253-276, 2005.

[16] R. P. Agarwal, M. Meehan, and D. O'Regan, Fixed Point Theory and Applications, Cambridge University Press, Cambridge, UK, 2001.

[17] Y. Liu, "Boundary value problems for second order differential equations on unbounded domains in a Banach space," Applied Mathematics and Computation, vol. 135, no. 2-3, pp. 569-584, 2003.

[18] Y. S. Liu, "Positive solutions of boundary value problems for nonlinear singular differential equations in Banach spaces," Acta Mathematica Sinica, vol. 47, no. 1, pp. 131-140, 2004.

[19] Y. Cui and Y. Zou, "Positive solutions of nonlinear singular boundary value problems in abstract spaces," Nonlinear Analysis: Theory, Methods \& Applications A, vol. 69, no. 1, pp. 287-294, 2008.

[20] J.-P. Aubin and I. Ekeland, Applied Nonlinear Analysis, John Wiley \& Sons, New York, NY, USA, 1984. 
[21] D. J. Guo, Nonlinear Functional Analysis, Shandong Science and Technology Publishing House, Jinan, China, 1985.

[22] H. Chen and P. Li, "Three-point boundary value problems for second-order ordinary differential equations in Banach spaces," Computers \& Mathematics with Applications, vol. 56, no. 7, pp. 1852-1860, 2008. 


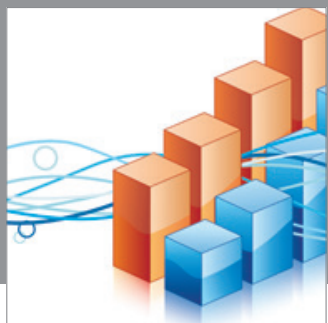

Advances in

Operations Research

mansans

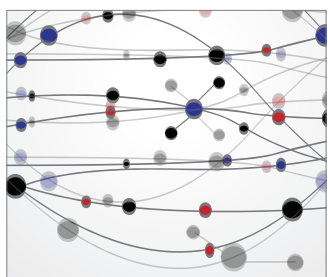

The Scientific World Journal
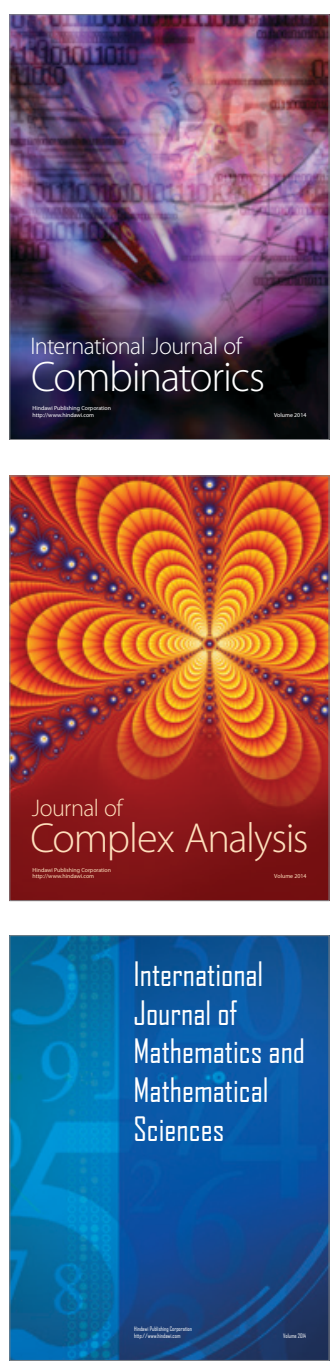
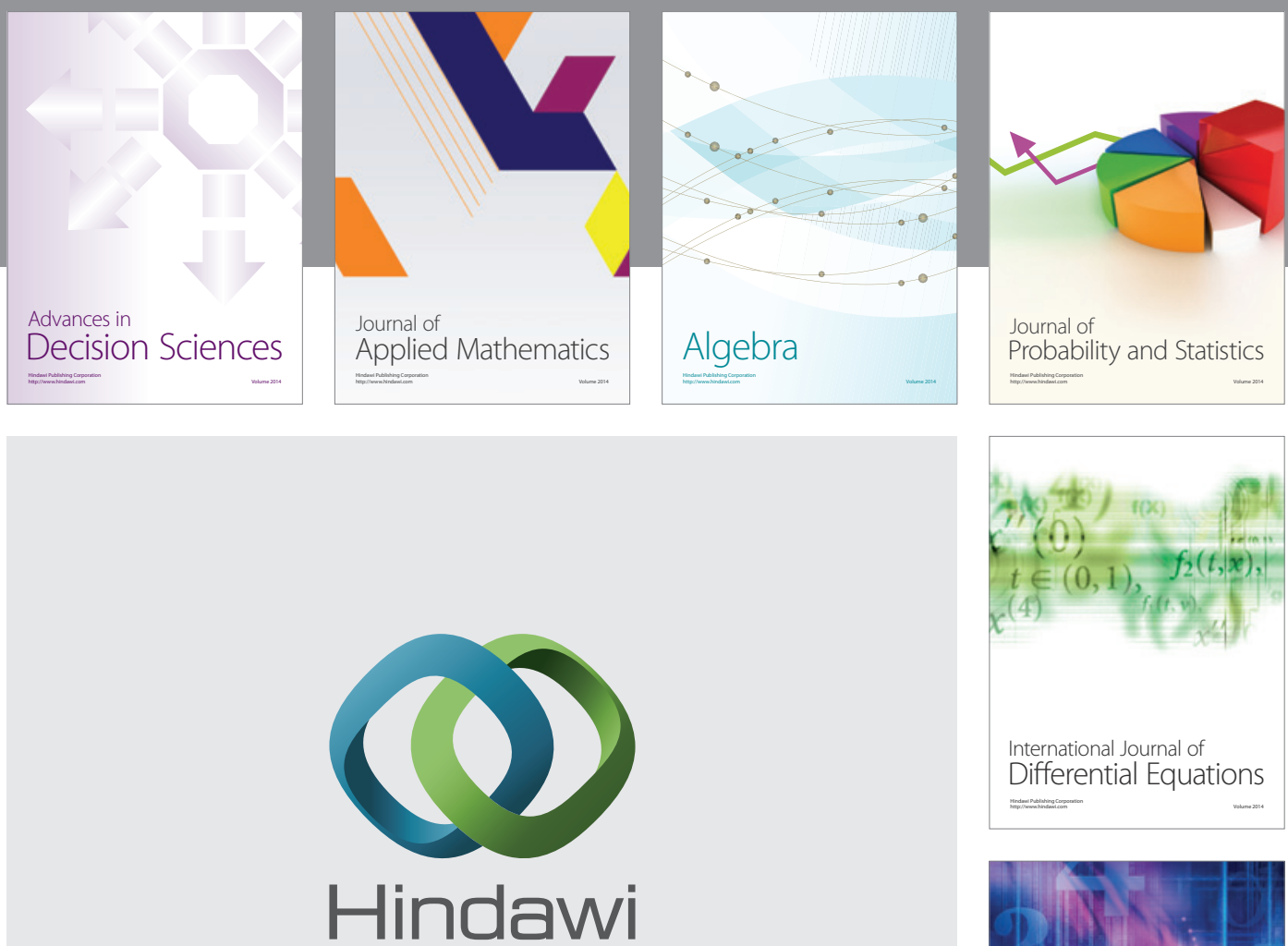

Submit your manuscripts at http://www.hindawi.com
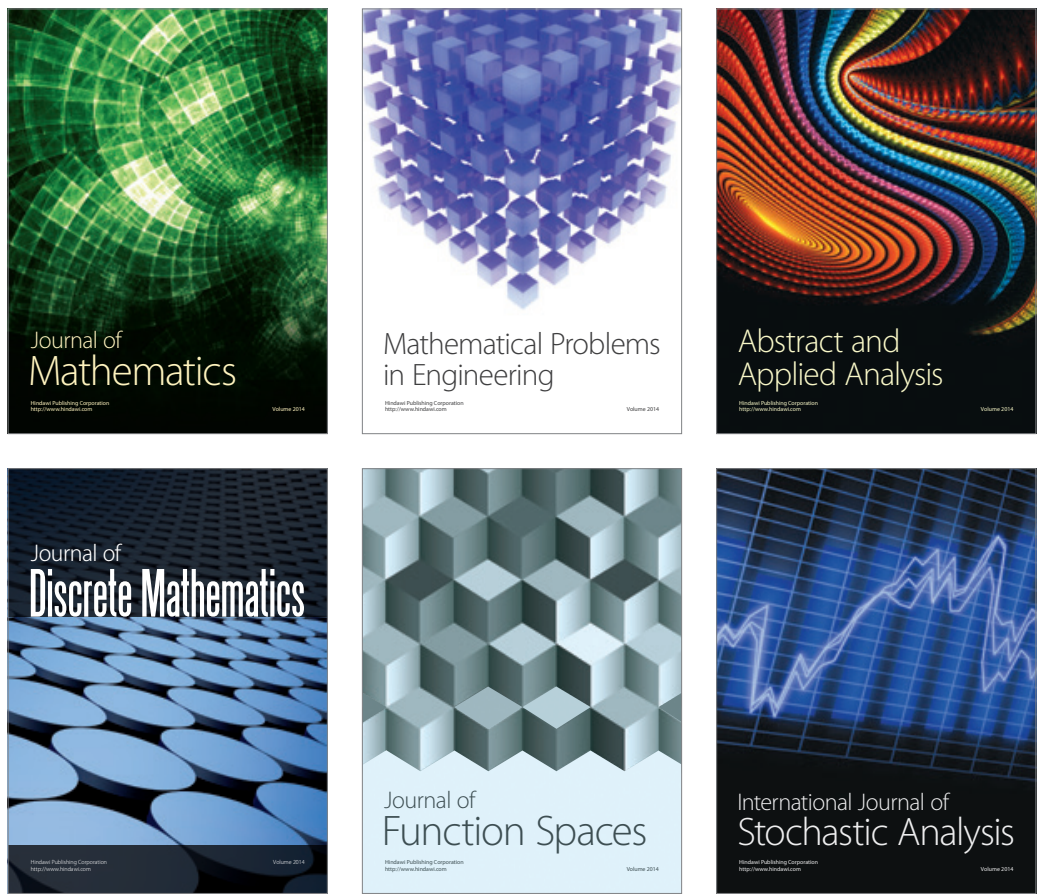

Journal of

Function Spaces

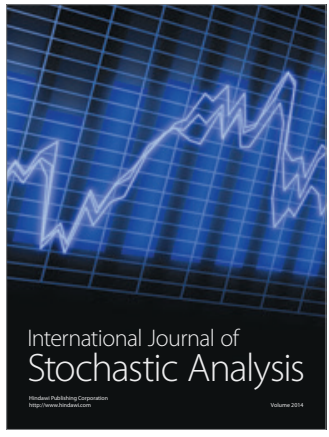

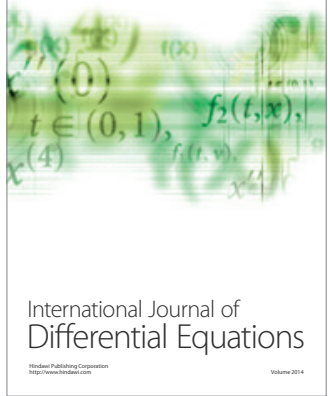
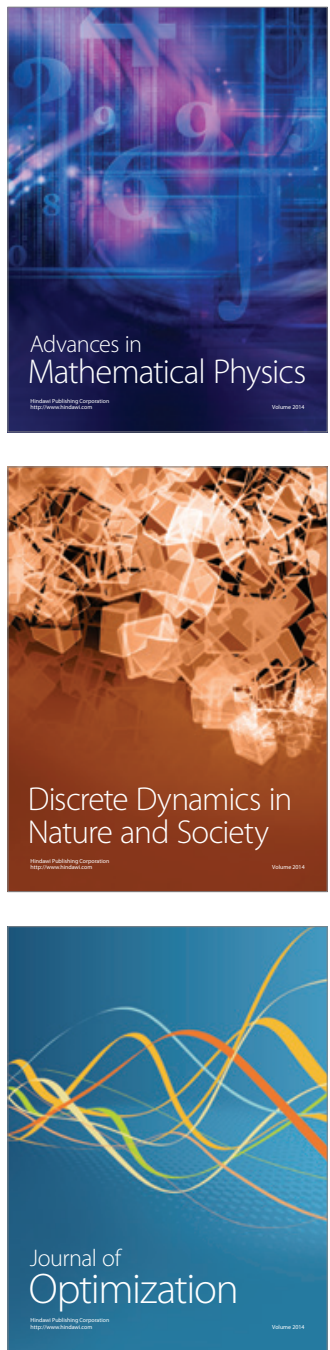